\title{
Inclusive electron-nucleus cross section within the self-consistent Green's function approach
}

\author{
N. Rocco and C. Barbieri \\ Department of Physics, University of Surrey, Guildford, GU2 7HX, United Kingdom
}

(Received 13 March 2018; revised manuscript received 11 June 2018; published 8 August 2018)

\begin{abstract}
We present results for charge form factors, the point-proton, charge, and single-nucleon momentum distributions of ${ }^{4} \mathrm{He}$ and ${ }^{16} \mathrm{O}$ obtained within the self-consistent Green's function approach. The removal of the center-of-mass contribution for both nuclei has been performed by using a METROPOLIS Monte Carlo algorithm in which the center-of-mass coordinate can be exactly subtracted from the optimal reference state wave function generated during the self-consistent Green's function calculations. The spectral functions of the same two nuclei have been used to compute inclusive electron-nucleus cross sections. The formalism adopted is based on the factorization of the spectral function and the nuclear transition matrix elements. This allows us to provide an accurate description of nuclear dynamics and to account for relativistic effects in the interaction vertex. When final-state interactions for the struck particle are accounted for, we find nice agreement between the data and the theory for the inclusive electron- ${ }^{16} \mathrm{O}$ cross section. The results lay the foundations for future applications of the self-consistent Green's function method, in both closed and open shell nuclei, to neutrino data analysis.
\end{abstract}

DOI: 10.1103/PhysRevC.98.025501

\section{INTRODUCTION}

The current and next generations of neutrino oscillation experiments require nuclear physics calculations of the structure and electroweak properties of atomic nuclei supplemented by quantified theoretical uncertainties [1-3]. The Deep Underground Neutrino Experiment (DUNE) will exploit liquid argon time-projection chambers (TPCs) to test charge parity (CP) violation in the lepton sector and to shed light on the neutrino mass hierarchy. Hence, nuclear theories able to tackle genuine open-shell nuclei, such as argon, will be critical to the reconstruction of the initial neutrino energy.

The self-consistent Green's function (SCGF) approach is an ab initio method in which the optical potential and spectral functions are calculated covering the full spectra of both nucleon attachment and removal (i.e., both close and far forms of the Fermi surface) [4-9]. The self-consistency feature means that the input information about the ground state and excitations of the systems no longer depends on a user-defined reference state but instead it is taken directly from the computed correlated propagator. The SCGF method has recently been reformulated within Gorkov's theory that allows it to address open-shell nuclei [10-12]. Within this approach, the description of pairing correlations characterizing open-shell systems is achieved by breaking particle number symmetry. The method was extended to include three-body interactions in Ref. [13,14]. Modern two- and three-nucleon chiral forces can be fully exploited within this formalism. Because of these features, SCGF theory is a prime tool for providing the nuclear structure input necessary to calculate electroweak properties of nuclei. However, its performance in predicting lepton-nucleus reactions with chiral nuclear forces is still to be assessed.

In this work, we use the saturating next-to-next-to-leadingorder (NNLO) interaction denoted as $\mathrm{NNLO}_{\text {sat }}$ [15] and calculate the SCGF spectral functions of ${ }^{4} \mathrm{He}$ and ${ }^{16} \mathrm{O}$. We obtain their point density, charge density, and single-momentum distribution. All calculations are performed expanding on an harmonic oscillator basis and the dependence of the results on the oscillator parameters is investigated. For light nuclei, such as ${ }^{4} \mathrm{He}$, spurious contributions of the center of mass in the calculated wave functions can be sizable in the model spaces exploited by SCGF and other post-Hartree-Fock methods. While it is possible to show that the center of mass effectively decouples from the relative motion for sufficiently large spaces [16], subtracting its effect from the calculated wave function and spectral functions is a long-standing nontrivial issue. Here, we address this problem by performing a Monte Carlo integration in which the center-of-mass component is exactly subtracted from the wave function.

In the high momentum transfer region of neutrino-nucleus scattering, the formalism based on spectral function and factorization of the nuclear transition matrix elements allows us to combine a fully relativistic description of the single-nucleon interaction vertex with an accurate treatment of nuclear dynamics $[17,18]$. In order to apply any theoretical model in the neutrino data analysis, it is fundamental to validate it against the large body of electron scattering data.

This work has to be considered as a first step in this direction. In fact, we present an extensive comparison with the experimental data of the electromagnetic double differential cross sections of ${ }^{4} \mathrm{He}$ and ${ }^{16} \mathrm{O}$ scattering, which was obtained by exploiting the corresponding SCGF spectral functions. The predictions for ${ }^{16} \mathrm{O}$ are important for the data analysis of Super-Kamiokande, in which a water Cherenkov detector to study neutrinos produced from different sources is used. A detailed analysis of theoretical uncertainties would also require studies of the dependence of the nuclear Hamiltonian at different resolution scales. This will be a subject of future work. 
In Sec. II, we briefly review the SCGF formalism and the links of propagators with the one-body density distribution and other quantities of experimental interest. Section III is devoted to the derivation of the electron-nucleus cross section within the impulse approximation (IA) in which the factorization of the nuclear transition matrix elements is assumed. Final-state interactions (FSI) involving the struck particle are treated as corrections. They are included using the convolution approach of Refs. $[19,20]$. In Sec. IV, we present results for the point density, charge density, and single-momentum distribution of ${ }^{4} \mathrm{He}$ and ${ }^{16} \mathrm{O}$. In addition, the inclusive electromagnetic cross sections of these two nuclei, obtained using the associated SCGF spectral functions, are compared with the experimental data and the role played by FSI is discussed. Conclusions are drawn in Sec. V.

\section{THE SELF-CONSISTENT GREEN'S FUNCTION APPROACH}

The one-body Green's function is written as a sum of two different contributions describing the propagation of a particle and hole state [21]:

$$
\begin{aligned}
g_{\alpha \beta}(\omega)= & \left\langle\psi_{0}^{A}\left|a_{\alpha} \frac{1}{\omega-\left(H-E_{0}^{A}\right)+i \eta} a_{\beta}^{\dagger}\right| \psi_{0}^{A}\right\rangle \\
& +\left\langle\psi_{0}^{A}\left|a_{\beta}^{\dagger} \frac{1}{\omega+\left(H-E_{0}^{A}\right)-i \eta} a_{\alpha}\right| \psi_{0}^{A}\right\rangle,
\end{aligned}
$$

where $\psi_{0}^{A}$ is the ground-state wave function of $A$ nucleons, and $a_{\alpha}^{\dagger}$ and $a_{\alpha}$ are the creation and annihilation operators in the quantum state $\alpha$, respectively. The so-called Lehmann representation results from inserting completeness relations in Eq. (1). This is

$$
\begin{aligned}
g_{\alpha \beta}(\omega)= & \sum_{n} \frac{\left\langle\psi_{0}^{A}\left|a_{\alpha}\right| \psi_{n}^{A+1}\right\rangle\left\langle\psi_{n}^{A+1}\left|a_{\beta}^{\dagger}\right| \psi_{0}^{A}\right\rangle}{\omega-\left(E_{n}^{A+1}-E_{0}^{A}\right)+i \eta} \\
& +\sum_{k} \frac{\left\langle\psi_{0}^{A}\left|a_{\beta}^{\dagger}\right| \psi_{k}^{A-1}\right\rangle\left\langle\psi_{k}^{A-1}\left|a_{\alpha}\right| \psi_{0}^{A}\right\rangle}{\omega-\left(E_{0}^{A}-E_{k}^{A-1}\right)-i \eta},
\end{aligned}
$$

where $\left|\psi_{n}^{A+1}\right\rangle\left(\left|\psi_{k}^{A-1}\right\rangle\right)$ are the eigenstates and $E_{n}^{A+1}\left(E_{k}^{A-1}\right)$ are the eigenvalues of the $(A \pm 1)$-body system. Introducing the transition amplitudes

$$
\begin{aligned}
\left(\mathcal{X}_{\alpha}^{n}\right)^{*} & =\left\langle\psi_{0}^{A}\left|a_{\alpha}\right| \psi_{n}^{A+1}\right\rangle, \\
\mathcal{Y}_{\alpha}^{k} & =\left\langle\psi_{k}^{A-1}\left|a_{\alpha}\right| \psi_{0}^{A}\right\rangle
\end{aligned}
$$

and the corresponding quasiparticle energies

$$
\begin{aligned}
& \epsilon_{n}^{+}=E_{n}^{A+1}-E_{0}^{A}, \\
& \epsilon_{k}^{-}=E_{0}^{A}-E_{k}^{A-1}
\end{aligned}
$$

leads to the more compact expression

$$
g_{\alpha \beta}(\omega)=\sum_{n} \frac{\left(\mathcal{X}_{\alpha}^{n}\right)^{*} \mathcal{X}_{\beta}^{n}}{\omega-\epsilon_{n}^{+}+i \eta}+\sum_{k} \frac{\mathcal{Y}_{\alpha}^{k}\left(\mathcal{Y}_{\beta}^{k}\right)^{*}}{\omega-\epsilon_{k}^{-}-i \eta} .
$$

The one-body propagator given in Eqs. (1) and (2) is completely determined by solving the Dyson equation

$$
g_{\alpha \beta}(\omega)=g_{\alpha \beta}^{0}(\omega)+\sum_{\gamma \delta} g_{\alpha \gamma}^{0}(\omega) \Sigma_{\gamma \delta}^{\star}(\omega) g_{\delta \beta}(\omega),
$$

where $g_{\alpha \beta}^{0}(\omega)$ is the unperturbed single-particle propagator and $\Sigma_{\gamma \delta}^{\star}(\omega)$ is the irreducible self-energy that encodes nuclear medium effects in the particle propagator [21]. The latter is given by the sum of two different terms,

$$
\Sigma_{\alpha \beta}^{\star}(\omega)=\Sigma_{\alpha \beta}^{\infty}+\tilde{\Sigma}_{\alpha \beta}(\omega),
$$

where the first one describes the average mean field and the second one contains dynamic correlations. In practical calculations, the self-energy is expanded as a function of the propagator itself, implying that an iterative procedure is required to solve the Dyson equation self-consistently. The selfenergy can be calculated systematically within the algebraic diagrammatic construction (ADC) method. The third-order truncation of this scheme [ADC(3)] yields a propagator that includes all possible Feynman contributions up to third order but it further resums infinite series of relevant diagrams in a nonperturbative fashion [22,23]. Two- and three-nucleon force contributions are included. A first organization of the contributions to the self-energy comes by considering the particle irreducible (PI) and skeleton diagrams. The number of Feynman diagrams entering the calculation of the Green's function rapidly increases when three- or many-body forces are accounted for. In order to circumvent this problem and reduce the number of Feynman diagrams to be considered, a useful strategy is to include only interaction-irreducible diagrams [13]. For our calculations, we use the following mediumdependent or effective one- and two-body interactions:

$$
\begin{aligned}
\tilde{U}_{\alpha \beta} & =U_{\alpha \beta}+\sum_{\delta \gamma} V_{\alpha \gamma, \beta \delta} \rho_{\delta \gamma}+\frac{1}{4} \sum_{\mu \nu \gamma \delta} W_{\alpha \mu \nu, \beta \gamma \delta} \rho_{\gamma \mu} \rho_{\delta \nu}, \\
\tilde{V}_{\alpha \beta, \gamma \delta} & =V_{\alpha \beta, \gamma \delta}+\sum_{\mu \nu} W_{\alpha \beta \mu, \gamma \delta \nu} \rho_{\nu \mu},
\end{aligned}
$$

where $U, V$, and $W$ label the matrix elements of the one-, two-, and three-body interactions, respectively. The one-body density matrix appearing in Eq. (8) reads

$$
\rho_{\delta \gamma}=\left\langle\psi_{0}^{A}\left|a_{\gamma}^{\dagger} a_{\delta}\right| \psi_{0}^{A}\right\rangle .
$$

The use of this averaging procedure allows us to retain only interaction-irreducible diagrams in the effective interactions $\tilde{U}$ and $\tilde{V}$, while residual contributions that include $W$ can be safely neglected [24-27]. The expressions of the static and dynamic self-energy up to third order, including all possible two- and three-nucleon terms that enter the expansion of the self-energy, as well as interaction-irreducible (i.e., not averaged) three-nucleon diagrams have been recently derived in Ref. [28].

Figure 1 displays the three simplest diagrams that enter the present calculation of the self-energy. These are taken as "seeds" for an all order resummation that eventually generates $\Sigma_{\alpha \beta}^{\star}(\omega)$. The first contribution is at second order while the last two are of third order in the expansion of Eq. (7). Note that for all the considered diagrams, the set of intermediate state 

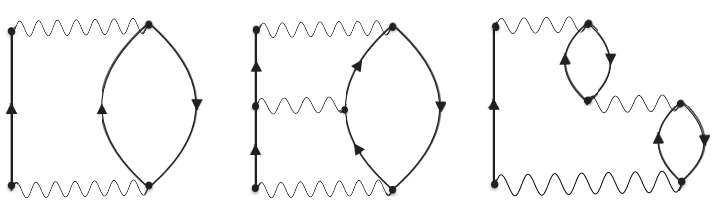

FIG. 1. One-particle irreducible skeleton and interaction irreducible diagrams with $2 \mathrm{p} 1 \mathrm{~h}$ intermediate configurations. The wiggly lines represent the two-body effective interaction of Eq. (8). The corresponding diagrams for the $2 \mathrm{~h} 1 \mathrm{p}$ intermediate configurations are obtained by flipping the orientation of the lines.

configurations corresponds to two-particle-one-hole (2p1h) and two-hole-one-particle ( $2 \mathrm{~h} 1 \mathrm{p})$ arrangements and that we use the two-nucleon effective interaction of Eq. (8). Within the ADC(3) approach, an infinite-order summation of diagrams of Fig. 1 that includes particle-particle and hole-hole ladders as well as particle-hole rings is performed. The dynamical part of the self-energy of Eq. (7) can be rewritten in the Lehmann representation as

$$
\tilde{\Sigma}_{\alpha \beta}(\omega)=\sum_{i j^{\prime}} \mathbf{D}_{\alpha i}^{\dagger}\left[\frac{1}{\omega-(\mathbf{K}+\mathbf{C})}\right]_{i j} \mathbf{D}_{j \beta}^{\dagger},
$$

where $\mathbf{K}$ are the unperturbed $2 \mathrm{p} 1 \mathrm{~h}$ and $2 \mathrm{~h} 1 \mathrm{p}$ energies, $\mathbf{D}$ coupling matrices, and $\mathbf{C}$ interaction matrices for the forward and backward intermediate states.

The calculations presented in this work have been performed expanding one-, two-, and three-body operators on a spherical harmonic oscillator basis whose dimension and the oscillation frequency are denoted by $N_{\max }=\max \{2 n+\ell\}$ and $\hbar \Omega$, respectively.

The point-proton density distribution can be readily obtained from Eq. (9) and reads

$$
\rho_{p}(\mathbf{r})=\sum_{\alpha \beta} \Phi_{\beta}^{*}(\mathbf{r}) \Phi_{\alpha}(\mathbf{r}) \rho_{\alpha \beta},
$$

where $\Phi_{\alpha}(\mathbf{r})=\langle\mathbf{r} \mid \alpha\rangle$ denotes the harmonic oscillator singeparticle wave functions and the sum includes only proton single-particle states. Analogous expressions can be written for point-neutron and matter density distributions.

The computational cost required to account for the fragmentation of the single-particle propagator into Eq. (10) rapidly increases with the size of the nucleus and of the model space. For this reason, an optimized reference state (OpRS) approach is used to approximate the single-particle propagators entering in the diagrams of Fig. 1 [8]. The OpRS is taken to be an independent particle model propagator as

$$
g_{\alpha \beta}^{\mathrm{OpRS}}=\sum_{n \notin F} \frac{\left(\phi_{\alpha}^{n}\right)^{*} \phi_{\beta}^{n}}{\omega-\epsilon_{n}^{\mathrm{OpRS}}+i \eta}+\sum_{k \in F} \frac{\phi_{\alpha}^{k}\left(\phi_{\beta}^{k}\right)^{*}}{\omega-\epsilon_{k}^{\mathrm{OpRS}}-i \eta},
$$

where $F$ represents the set of occupied states and $\epsilon^{\mathrm{OpRS}}$ and $\phi$ are the single-particle energies and wave functions, respectively. The OpRS propagator is chosen to best approximate the correlated one while keeping a reduced number of poles. This is achieved by introducing the following moments of the spectral distribution with respect to energy poles:

$$
\begin{aligned}
& M_{\alpha \beta}^{0}=\sum_{n}\left(\mathcal{X}_{\alpha}^{n}\right)^{*} \mathcal{X}_{\beta}^{n}+\sum_{k} \mathcal{Y}_{\alpha}^{k}\left(\mathcal{Y}_{\beta}^{k}\right)^{*}, \\
& M_{\alpha \beta}^{1}=\sum_{n} \frac{\left(\mathcal{X}_{\alpha}^{n}\right)^{*} \mathcal{X}_{\beta}^{n}}{\left(E_{F}-\epsilon_{n}^{+}\right)}+\sum_{k} \frac{\mathcal{Y}_{\alpha}^{k}\left(\mathcal{Y}_{\beta}^{k}\right)^{*}}{\left(E_{F}-\epsilon_{k}^{-}\right)},
\end{aligned}
$$

where $E_{F}=\left(\epsilon_{0}^{+}+\epsilon_{0}^{-}\right) / 2=\left(E_{0}^{A+1}-E_{0}^{A-1}\right) / 2$ and the transition amplitudes are given in Eqs. (3). The quantities in Eq. (13) are important since they constrain the density distributions, one-body observables and the Koltun energy sum rule of the propagator [29]. Hence, we obtain $\epsilon^{\mathrm{OpRS}}$ and $\phi$ by requiring that the OpRS lowest momenta of the spectral distribution reproduce those of the full calculation, i.e., $M_{\alpha \beta}^{0, O p R S}=M_{\alpha \beta}^{0}$ and $M_{\alpha \beta}^{1, O p R S}=M_{\alpha \beta}^{1}$ [8].

The elastic scattering of a nucleus hit by a probe and recoiling with a momentum $\mathbf{q}$ is described by the elastic form factor $F_{L}(\mathbf{q})$. Neglecting the small spin-orbit contribution, the latter is given by

$$
F_{L}(\mathbf{q})=\frac{1}{Z}\left\langle\psi_{0}^{A}\left|\left[\frac{\sum_{i} \epsilon_{i}}{\sqrt{1+Q_{e l}^{2} /\left(4 m^{2}\right)}}\right]\right| \psi_{0}^{A}\right\rangle,
$$

where in the laboratory frame $Q_{e l}^{2}=|\mathbf{q}|^{2}-w_{e l}^{2}, \quad \omega_{e l}=$ $\sqrt{|\mathbf{q}|^{2}+m_{A}^{2}}-m_{A}$ is the energy transfer corresponding to the elastic scattering, $m_{A}$ is the target nucleus mass, and

$$
\epsilon_{i}=G_{E}^{p}\left(Q_{e l}^{2}\right) \frac{\left(1+\tau_{z_{i}}\right)}{2}+G_{E}^{n}\left(Q_{e l}^{2}\right) \frac{\left(1-\tau_{z_{i}}\right)}{2}
$$

with $G_{E}^{p}\left(Q_{e l}^{2}\right)$ and $G_{E}^{n}\left(Q_{e l}^{2}\right)$ being the proton and neutron electric form factors, respectively. The elastic form factor can be rewritten in terms of the Fourier transforms of the point-proton and nucleon densities as

$$
F_{L}(\mathbf{q})=\frac{1}{Z} \frac{G_{E}^{p}\left(Q_{e l}^{2}\right) \tilde{\rho}_{p}(q)+G_{E}^{n}\left(Q_{e l}^{2}\right) \tilde{\rho}_{n}(q)}{\sqrt{1+Q_{e l}^{2} /\left(4 m^{2}\right)}},
$$

where

$$
\begin{aligned}
\tilde{\rho}_{p, n}(q)= & \int d^{3} r_{1} \ldots d^{3} r_{A} e^{i \mathbf{q} \cdot \mathbf{r}_{1}} \psi_{0}^{*}\left(r_{1}, \ldots, r_{A}\right) \\
& \times \frac{1 \pm \tau_{z_{1}}}{2} \psi_{0}\left(r_{1}, \ldots, r_{A}\right) \\
= & \int d^{3} r e^{i \mathbf{q} \cdot \mathbf{r}} \rho_{p, n}(\mathbf{r}) .
\end{aligned}
$$

Note that the factor $\sqrt{1+Q_{e l}^{2} /\left(4 m^{2}\right)}$ in the denominator of Eq. (16) accounts for relativistic corrections to the charge operator. Assuming that $\tilde{\rho}_{p}(q)=\tilde{\rho}_{n}(q)$, the charge distribution can be written as

$$
\begin{aligned}
\rho_{c h}\left(r^{\prime}\right) & =\int \frac{d^{3} q}{(2 \pi)^{3}} e^{-i \mathbf{q} \cdot \mathbf{r}^{\prime}} F_{L}(\mathbf{q}) \\
& =\int \frac{d^{3} q}{(2 \pi)^{3}} e^{-i \mathbf{q} \cdot \mathbf{r}^{\prime}} \frac{1}{Z} \frac{\left[G_{E}^{p}\left(Q_{e l}^{2}\right)+G_{E}^{n}\left(Q_{e l}^{2}\right)\right] \tilde{\rho}_{p}(q)}{\sqrt{1+Q_{e l}^{2} /\left(4 m^{2}\right)}} .
\end{aligned}
$$


The probability of finding a nucleon in the nucleus with momentum $\mathbf{k}$ is proportional to its momentum distribution. The latter can be written in terms of the one-body density matrix of Eq. (9) as

$$
n(\mathbf{k})=\sum_{\alpha \beta} \tilde{\Phi}_{\beta}^{*}(\mathbf{k}) \tilde{\Phi}_{\alpha}(\mathbf{k}) \rho_{\alpha \beta}
$$

where $\tilde{\Phi}_{\alpha}(\mathbf{k})$ is the Fourier transform of the harmonic oscillator wave function

$$
\tilde{\Phi}_{\alpha}(\mathbf{k})=\int d^{3} r e^{i \mathbf{k} \mathbf{r}} \Phi_{\alpha}(\mathbf{r})
$$

The momentum distribution is normalized as $\int d^{3} k n(\mathbf{k}) /(2 \pi)^{3}=\mathcal{N}$, with $\mathcal{N}$ being the number of either protons $Z$ or neutrons $(A-Z)$.

The subtraction of the center-of-mass contribution from the wave function is a long-standing issue affecting a number of nuclear many-body approaches relying on a single-nucleon basis expansions. While for medium and heavy nuclei this correction can be safely neglected, the center-of-mass contribution strongly affects the results of light nuclei, such as ${ }^{4} \mathrm{He}$. In order to address this problem, we developed a METROPOLIS Monte Carlo (MMC) code, analogous to the one used in variational Monte Carlo studies [30], that allows us to single out the centerof-mass contribution to the wave function in the calculation of the charge density and the momentum distribution. The wave function we used for the MMC is the Slater determinant obtained from the OpRS calculation, $\left|\psi_{V}\right\rangle=\left|\psi_{0}^{O p R S}\right\rangle$. At variance with the fully correlated propagator of Eq. (5), the use of the unperturbed $g$ OpRS (i.e., a Slater determinant) allows for a unique definition of the wave function. The spatial integrals of Eqs. (17), (19), and (20) have been performed using METROPOLIS Monte Carlo techniques [31]. A sequence of points in the $3 A$-dimensional space denoted by $\mathbf{R}=\left\{\mathbf{r}_{1}, \ldots, \mathbf{r}_{A}\right\}$ are generated by sampling from the probability distribution

$$
P(\mathbf{R})=\left|\psi_{0}^{O p R S}(\mathbf{R})\right|^{2} .
$$

At each step of the calculation, the center-of-mass contribution to the wave function is subtracted, computing the wave function and the expectation value in the intrinsic coordinates given by

$$
\tilde{\mathbf{r}}_{i}=\mathbf{r}_{i}-\mathbf{R}_{\mathrm{c} . \mathrm{m} .}, \quad \mathbf{R}_{\mathrm{c} . \mathrm{m} .}=\frac{1}{A} \sum_{i} \mathbf{r}_{i} .
$$

Hence, the identification of the intrinsic contribution is easily achieved within quantum Monte Carlo (QMC) algorithms [32-35], since we always have access to the set of $3 A$ coordinates of the constituent nucleons.

\section{THE IMPULSE APPROXIMATION AND CONVOLUTION APPROACH}

In the one-photon-exchange approximation, the double differential electron-nucleus cross section takes the form

$$
\frac{d^{2} \sigma}{d E_{e^{\prime}} d \Omega_{e^{\prime}}}=\frac{\alpha^{2}}{q^{4}} \frac{E_{e^{\prime}}}{E_{e}} L_{\mu \nu} W^{\mu \nu},
$$

where $k_{e}=\left(E_{e}, \mathbf{k}_{e}\right)$ and $k_{e^{\prime}}=\left(E_{e^{\prime}}, \mathbf{k}_{e^{\prime}}\right)$ are the laboratory fourmomenta of the incoming and outgoing electrons, respectively; $\alpha \simeq 1 / 137$ is the fine structure constant, $d \Omega_{e^{\prime}}$ is the differential solid angle in the direction of $\mathbf{k}_{e^{\prime}}$, and $q=k_{e}-k_{e^{\prime}}=(\omega, \mathbf{q})$ is the four-momentum transfer. The leptonic tensor is given by

$$
L^{\mu v}=2\left(k_{e^{\prime}}^{\mu} k_{e}^{v}+k_{e}^{\mu} k_{e^{\prime}}^{\nu}-g^{\mu \nu} k_{e^{\prime}} k_{e}\right) .
$$

The hadronic tensor is written in terms of matrix elements of the nuclear current operator between the target ground state and the hadronic final states as

$$
\begin{aligned}
W^{\mu \nu}= & \sum_{f}\left\langle\psi_{0}^{A}\left|J^{\mu^{\dagger}}(q)\right| \psi_{f}^{A}\right\rangle\left\langle\psi_{f}^{A}\left|J^{\nu}(q)\right| \psi_{0}^{A}\right\rangle \\
& \times \delta^{(4)}\left(P_{0}+q-P_{f}\right),
\end{aligned}
$$

where $\left|\psi_{0}^{A}\right\rangle$ and $\left|\psi_{f}^{A}\right\rangle$ denote the initial and final hadronic states with four-momenta $P_{0}=\left(E_{0}, \mathbf{p}_{0}\right)$ and $P_{f}=\left(E_{f}, \mathbf{p}_{f}\right)$, while $J(q)$ is the electromagnetic nuclear current operator.

At relatively large momentum transfer, $|\mathbf{q}| \gtrsim 500 \mathrm{MeV}$, the impulse approximation (IA) can be safely applied. Within this approximation, the interaction between the struck nucleon and the spectator $(A-1)$ particles is neglected $[18,36]$. The nuclear current operator reduces to a sum of one-body terms, $J(q)=\sum_{i} j_{i}(q)$, and the hadronic final state factorizes as

$$
|f\rangle \rightarrow|p\rangle \otimes\left|\psi_{f}^{A-1}\right\rangle \text {. }
$$

In the above equation, $|p\rangle$ denotes the final-state nucleon with momentum $\mathbf{p}$, while $\left|\psi_{f}^{A-1}\right\rangle$ describes the $(A-1)$-body spectator system. Its energy and recoiling momentum are fixed by energy and momentum conservation, yielding

$$
E_{f}^{A-1}=\omega+E_{0}^{A}-e(\mathbf{p}), \quad \mathbf{P}_{f}^{A-1}=\mathbf{q}-\mathbf{p} .
$$

Using the factorization ansatz and inserting a single-nucleon completeness relation, the matrix element of the current operator can be written as

$$
\left\langle\psi_{0}^{A}\left|J^{\mu}\right| \psi_{f}^{A}\right\rangle \rightarrow \sum_{k}\left\langle\psi _ { 0 } ^ { A } \left|\left[|k\rangle \otimes\left|\psi_{f}^{A-1}\right\rangle\right]\left\langle k\left|\sum_{i} j_{i}^{\mu}\right| p\right\rangle .\right.\right.
$$

Substituting the last equation in Eq. (25), the contribution to the hadron tensor is given by

$$
\begin{aligned}
W^{\mu v}(\mathbf{q}, \omega)= & \sum_{p, k} \sum_{f, i}\left\langle k\left|j_{i}^{\mu \dagger}\right| p\right\rangle\left\langle p\left|j_{i}^{v}\right| k\right\rangle \\
& \times\left\langle\psi _ { 0 } ^ { A } \left|[ | \psi _ { f } ^ { A - 1 } \rangle \otimes | k \rangle ] \left[\left\langle\psi_{f}^{A-1}|\otimes\langle k|] \mid \psi_{0}^{A}\right\rangle\right.\right.\right. \\
& \times \delta\left(\omega-e(\mathbf{p})-E_{f}^{A-1}+E_{0}^{A}\right) .
\end{aligned}
$$

Momentum conservation in the single-nucleon vertex implies $\mathbf{p}=\mathbf{k}+\mathbf{q}$. The one-body current operator can be written as

$$
j_{i}^{\mu}=\left[F_{1 i} \gamma^{\mu}+i \frac{F_{2 i}}{2 m} \sigma^{\mu v} q_{v}\right]
$$

where

$$
F_{1,2 i}=\frac{\left(F_{1,2}^{S}+F_{1,2}^{V} \tau_{z i}\right)}{2},
$$

and $F^{S(V)}=F^{p} \pm F^{n}$. The latter are defined in terms of the electric and magnetic form factors via

$$
\begin{aligned}
& F_{1}^{S}=\frac{G_{E}^{S}+\tau G_{M}^{S}}{1+\tau}, \\
& F_{2}^{S}=\frac{G_{M}^{S}-G_{E}^{S}}{1+\tau},
\end{aligned}
$$


where $\tau=-q^{2} /\left(4 m^{2}\right)$. Finally, using the identity

$$
\begin{aligned}
& \delta\left(\omega-e(\mathbf{p})-E_{f}^{A-1}+E_{0}^{A}\right) \\
& \quad=\int d E \delta(\omega+E-e(\mathbf{p})) \delta\left(E+E_{f}^{A-1}-E_{0}^{A}\right),
\end{aligned}
$$

we can rewrite the hadron tensor as

$$
\begin{aligned}
W^{\mu \nu}(\mathbf{q}, \omega)= & \int \frac{d^{3} k}{(2 \pi)^{3}} d E P_{h}(\mathbf{k}, E) \frac{m^{2}}{e(\mathbf{k}) e(\mathbf{k}+\mathbf{q})} \\
& \times \sum_{i}\left\langle k\left|j_{i}^{\mu \dagger}\right| k+q\right\rangle\left\langle k+q\left|j_{i}^{v}\right| k\right\rangle \\
& \times \delta(\omega+E-e(\mathbf{k}+\mathbf{q})),
\end{aligned}
$$

where the factors $m / e(\mathbf{k})$ and $m / e(\mathbf{k}+\mathbf{q})$ have to be included to account for the implicit covariant normalization of the fourspinors of the initial and final nucleons in the matrix elements of the relativistic current.

The hole spectral function

$$
\begin{aligned}
P_{h}(\mathbf{k}, E)= & \sum_{f} \mid\left\langle\psi_{0}^{A}\left|\left[|k\rangle \otimes\left|\psi_{f}^{A-1}\right\rangle\right]\right|^{2}\right. \\
& \times \delta\left(E+E_{f}^{A-1}-E_{0}^{A}\right)
\end{aligned}
$$

gives the probability distribution of removing a nucleon with momentum $\mathbf{k}$ from the target nucleus, leaving the residual $(A-1)$ system with an excitation energy $E$. Note that in Eq. (34) we neglected Coulomb interactions and the other (small) isospin-breaking terms and made the assumption, largely justified in the case of closed-shell nuclei, that the proton and neutron spectral functions are identical.

Rewriting the nuclear matrix element as

$$
\begin{aligned}
{\left[\left\langle\psi_{f}^{A-1}|\otimes\langle k|] \mid \psi_{0}^{A}\right\rangle\right.} & =\sum_{\alpha} \mathcal{Y}_{\alpha}^{k} \tilde{\Phi}_{\alpha}(\mathbf{k}) \\
& =\sum_{\alpha} \tilde{\Phi}_{\alpha}(\mathbf{k})\left\langle\psi_{f}^{A-1}\left|a_{\alpha}\right| \psi_{0}^{A}\right\rangle,
\end{aligned}
$$

we recover the more familiar expression of the spectral function written as the imaginary part of the Green's function describing the propagation of a hole state

$$
\begin{aligned}
P_{h}(\mathbf{k}, E)= & \frac{1}{\pi} \sum_{\alpha \beta} \tilde{\Phi}_{\beta}^{*}(\mathbf{k}) \tilde{\Phi}_{\alpha}(\mathbf{k}) \\
& \times \operatorname{Im}\left\langle\psi_{0}^{A}\left|a_{\beta}^{\dagger} \frac{1}{E+\left(H-E_{0}^{A}\right)-i \epsilon} a_{\alpha}\right| \psi_{0}^{A}\right\rangle .
\end{aligned}
$$

In the kinematical region in which the interactions between the struck particle and the spectator system cannot be neglected, the IA results have to be modified to include the effect of FSI. Following Refs. [19,20], the multiple scatterings that the struck particle undergoes during its propagation through the nuclear medium are taken into account through a convolution scheme. The IA responses are folded with a function $f_{\mathbf{k}+\mathbf{q}}$, normalized as

$$
\int_{-\infty}^{+\infty} d \omega f_{\mathbf{k}+\mathbf{q}}(\omega)=1
$$

The double differential cross section is then given by

$$
\begin{aligned}
\left(\frac{d^{2} \sigma}{d E_{e^{\prime}} d \Omega_{e^{\prime}}}\right)_{F S I}= & \int \frac{d^{3} k}{(2 \pi)^{3}} d E \int d \omega^{\prime} f_{\mathbf{k}+\mathbf{q}}\left(\omega-\omega^{\prime}\right) \\
& \times \frac{m}{e(\mathbf{k})} \frac{m}{e(\mathbf{k}+\mathbf{q})} P_{h}(\mathbf{k}, E) \frac{\alpha^{2}}{q^{4}} \frac{E_{e^{\prime}}}{E_{e}} \\
& \times L_{\mu \nu} \sum_{i}\left\langle k\left|\left(j_{i}^{\mu}\right)^{\dagger}\right| k+q\right\rangle\left\langle k+q\left|j_{i}^{\nu}\right| k\right\rangle \\
& \times \delta\left(\omega^{\prime}+E-\tilde{e}(\mathbf{k}+\mathbf{q})\right) \theta\left(|\mathbf{k}+\mathbf{q}|-p_{F}\right) .
\end{aligned}
$$

In the last equation, we modified the energy spectrum of the struck nucleon

$$
\tilde{e}(\mathbf{k}+\mathbf{q})=e(\mathbf{k}+\mathbf{q})+U\left(t_{\mathrm{kin}}(\mathbf{k}+\mathbf{q})\right)
$$

by considering the real part of the optical potential $U$ derived from the Dirac phenomenological fit of Ref. [37]. This allows to describe the propagation of the knocked-out particle in the mean-field generated by the spectator system.

\section{RESULTS}

Our calculations have been performed using the $\mathrm{NNLO}_{\text {sat }}$ chiral interaction [15], which was specifically designed to accurately describe both binding energies and nuclear radii of midmass nuclei $[38,39]$. In Fig. 2 we analyze the convergence of the SCGF-ADC(3) point-proton densities of ${ }^{4} \mathrm{He}$ with respect to the oscillator frequency $(\hbar \Omega)$ and the size of the model space $\left(N_{\max }\right)$. The different lines almost superimpose, indicating that for $\hbar \Omega \approx 20 \mathrm{MeV}$ and $N_{\max } \geqslant 11$ the calculation converges and no longer depends on the oscillator parameters. The density calculated from the OpRS is also displayed. The nice agreement with the SCGF-ADC(3) curves follows from the requirement that the overlap functions in the

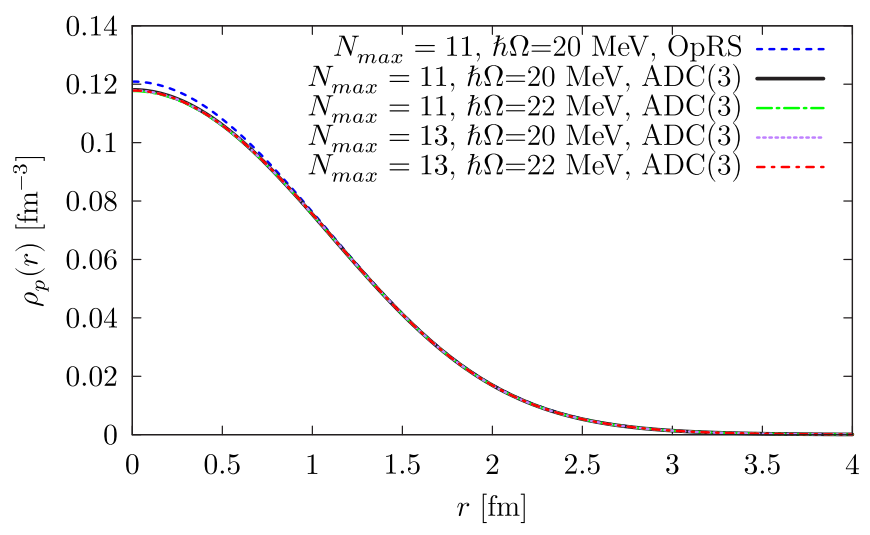

FIG. 2. Point proton densities in ${ }^{4} \mathrm{He}$, as predicted by $\mathrm{NNLO}_{\text {sat }}$. The dashed (blue) line corresponds to the OpRS derived for $N_{\max }=$ 11 and $\hbar \Omega=20 \mathrm{MeV}$. The other lines have been obtained using the SCGF full propagator for $N_{\max }=11,13$ and $\hbar \Omega=20,22 \mathrm{MeV}$. 


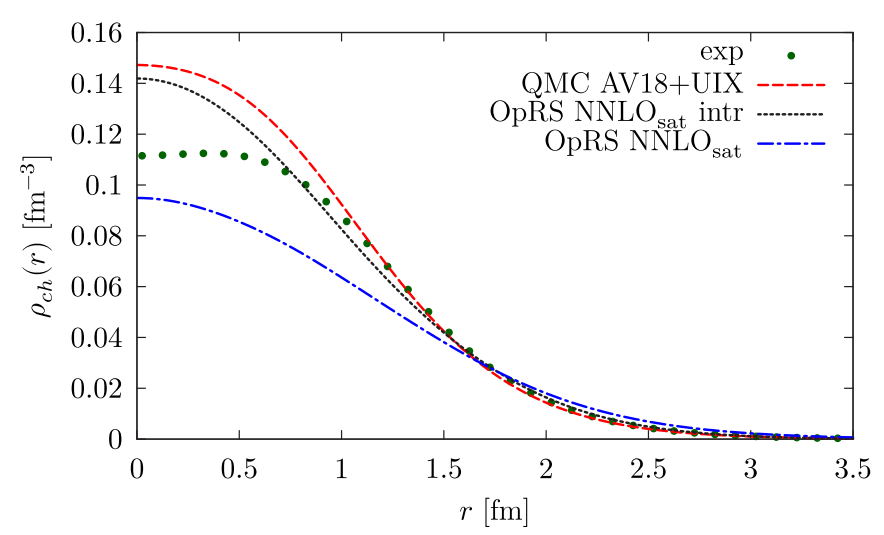

FIG. 3. Charge densities of ${ }^{4} \mathrm{He}$. The (green) dots have been obtained using the "sum-of-Gaussians" parametrization of the charge densities given in Ref. [40]. The dashed (red) line refers to the QMC calculation of Ref. [41] that used the AV18+UIX two- and three-body interactions. The dot-dashed (blue) line corresponds to the same OpRS propagator shown in Fig. 2, while in the short-dashed (black) line the center-of-mass contamination has been subtracted from the OpRS wave function by means a MMC calculation.

OpRS propagator are chosen to approximate at best the true (correlated) one-body density.

The charge densities in ${ }^{4} \mathrm{He}$ can be obtained from the point-proton densities through Eqs. (17) and (18). In Fig. 3, we compare the experimental charge density determined through the "sum of Gaussians" parametrization given in Ref. [40] with those obtained from the QMC results of Ref. [41] and from the OpRS calculated in the present work. For the latter, we display both the result already shown in Fig. 2 and the distribution obtained after subtracting the center-of-mass effect with the MMC algorithm outlined in Sec. II. When the center-ofmass contamination is subtracted, we obtain the short-dashed (black) line. The comparison with the total OpRS results, corresponding to the dot-dashed (blue) line, clearly shows that for ${ }^{4} \mathrm{He}$ the center-of-mass contribution is sizable and cannot be neglected. The use of the intrinsic wave function yields a strong enhancement of the charge density, which turns out to be very close to the QMC result. Note that the discrepancy between the experiment and the intrinsic OpRS and QMC calculations is motivated by the absence of the two-body meson exchange current contributions. These are known to have little effect on larger nuclei such as ${ }^{16} \mathrm{O}$ but their inclusion is fundamental in order to correctly reproduce the ${ }^{4} \mathrm{He}$ elastic form factor, from which the charge densities are extracted [30,41-43].

In Fig. 4, we compare the results for the charge elastic form factor for ${ }^{4} \mathrm{He}$ obtained within three many-body approaches in which different interactions have been used. It is visible that up to $q=3 \mathrm{fm}^{-1}$ the removal of the center-of-mass contamination enhances the strength and improves the agreement between the OpRS and the QMC and the calculations of Ref. [44]. For larger values of the momentum, we found some discrepancies in both the OpRS calculations.

For medium-mass nuclei, the center-of-mass corrections turn out to be less significant. This is clearly visible in Fig. 5 where we compare the full charge SCGF density calculated at the ADC(3) level with the intrinsic OpRS calculation. The

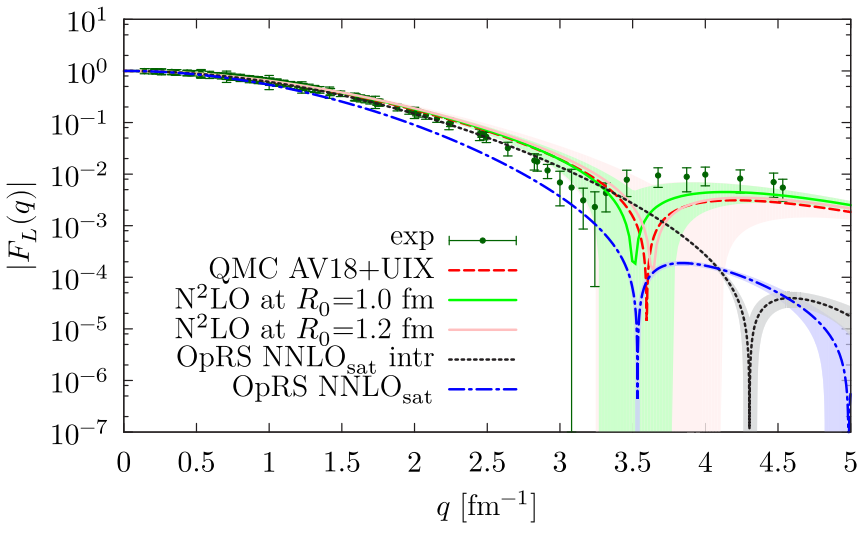

FIG. 4. Charge elastic form factor for ${ }^{4} \mathrm{He}$. The solid (light green and pink) lines correspond to the calculation of Ref. [44] where chiral two- and three-body interactions at $\mathrm{N}^{2} \mathrm{LO}$ have been used for $R_{0}=1.0 \mathrm{fm}$ and $R_{0}=1.2 \mathrm{fm}$ coordinate-space cutoffs, respectively. The uncertainty bands include the statistical MC uncertainties added in quadrature to the uncertainty from the truncation of the chiral expansion. The dashed (red) line is obtained within QMC Ref. [44] while the dot-dashed (blue) and short-dashed (black) line refers to the OpRS calculation with and without the center-of-mass contamination. The shaded area indicates the statistical MC uncertainty. Experimental data are from an unpublished compilation by Sick, based on Refs. [45-48].

construction of the ${ }^{16} \mathrm{O}$ wave function is more complicated than in the ${ }^{4} \mathrm{He}$ case where only the $s$-shell orbital has to be accounted for in the OpRS approximation. For this reason, the subtraction of the center-of-mass contribution required the use of a more sophisticated MC code. The charge density distribution obtained using the total and the intrinsic wave functions slightly differs and there is an overall nice agreement also with the QMC calculations. Both the SCGF-ADC(3) and the OpRS intrinsic results correctly reproduce the experimental points, confirming the goodness of the $\mathrm{NNLO}_{\text {sat }}$ potential, which was fitted to reproduce the experimental radius of ${ }^{16} \mathrm{O}$.

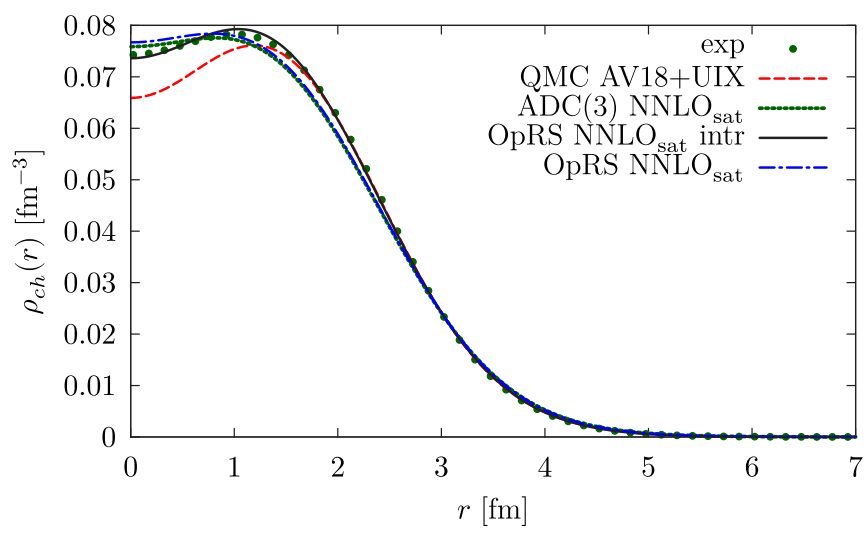

FIG. 5. Charge densities in ${ }^{16} \mathrm{O}$. The (green) dots and the dashed (red) line are the same as Fig. 3. The short-dashed (green) line corresponds to the full SCGF density calculated at the ADC(3) level, while the solid (black) and dot-dashed (blue) line refers to the OpRS calculation with and without the center-of-mass contamination, respectively. 


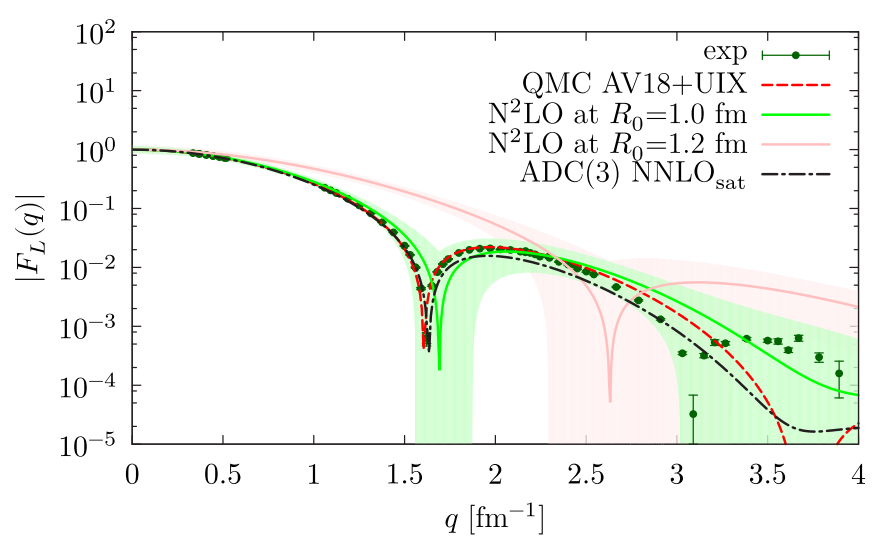

FIG. 6. Charge elastic form factor for ${ }^{16} \mathrm{O}$. The solid (light green) and (violet) lines correspond to the calculation of Ref. [43] for $R_{0}=1.0 \mathrm{fm}$ and $R_{0}=1.2 \mathrm{fm}$ coordinate-space cutoffs, respectively. The uncertainty bands include the statistical MC uncertainties added in quadrature to the uncertainty from the truncation of the chiral expansion. The dashed (red) line is obtained within QMC Ref. [43] while the dot-dashed (black) refers to the SCGF results calculated at the $\mathrm{ADC}(3)$ level. The shaded area indicates the statistical MC uncertainty. Experimental data are from Ref. [40].

Figure 6 displays the charge elastic form factor for ${ }^{16} \mathrm{O}$. In this case, we find an excellent agreement between the SCGF, the QMC calculations, and the experimental data. The results of Ref. [43] for two different values of the coordinate cutoffs are also shown.

In Fig. 7, we benchmark the intrinsic and uncorrected OpRS single-nucleon momentum distribution of ${ }^{4} \mathrm{He}$ with the QMC calculation of Ref. [41]. The OpRS result, corresponding to the dashed (blue) line, correctly follows that of the dressed ADC(3) propagator, although the agreement is not as close as in Fig. 2. Note that also in this case the subtraction of the center-of-mass component has a sizable effect, which is crucial for recovering the agreement with the intrinsic QMC results.

The ${ }^{16} \mathrm{O}$ single-nucleon momentum distributions obtained within the SCGF-ADC(3) and QMC approach are compared

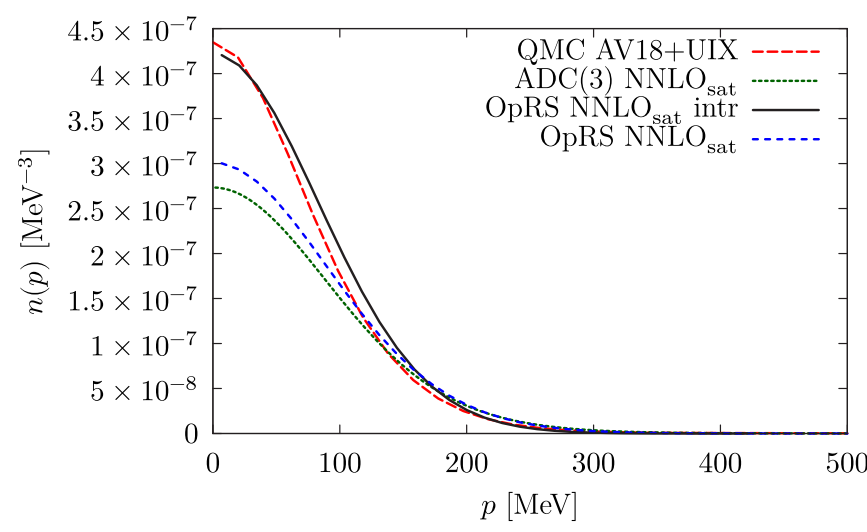

FIG. 7. Momentum distributions of ${ }^{4} \mathrm{He}$. The dashed (red) line corresponds to the QMC calculation [41], the dotted (green) curve has been obtained using the SCGF-ADC(3) propagator, while the short-dashed (blue) and solid (black) lines correspond to the total and intrinsic OpRS results, respectively.
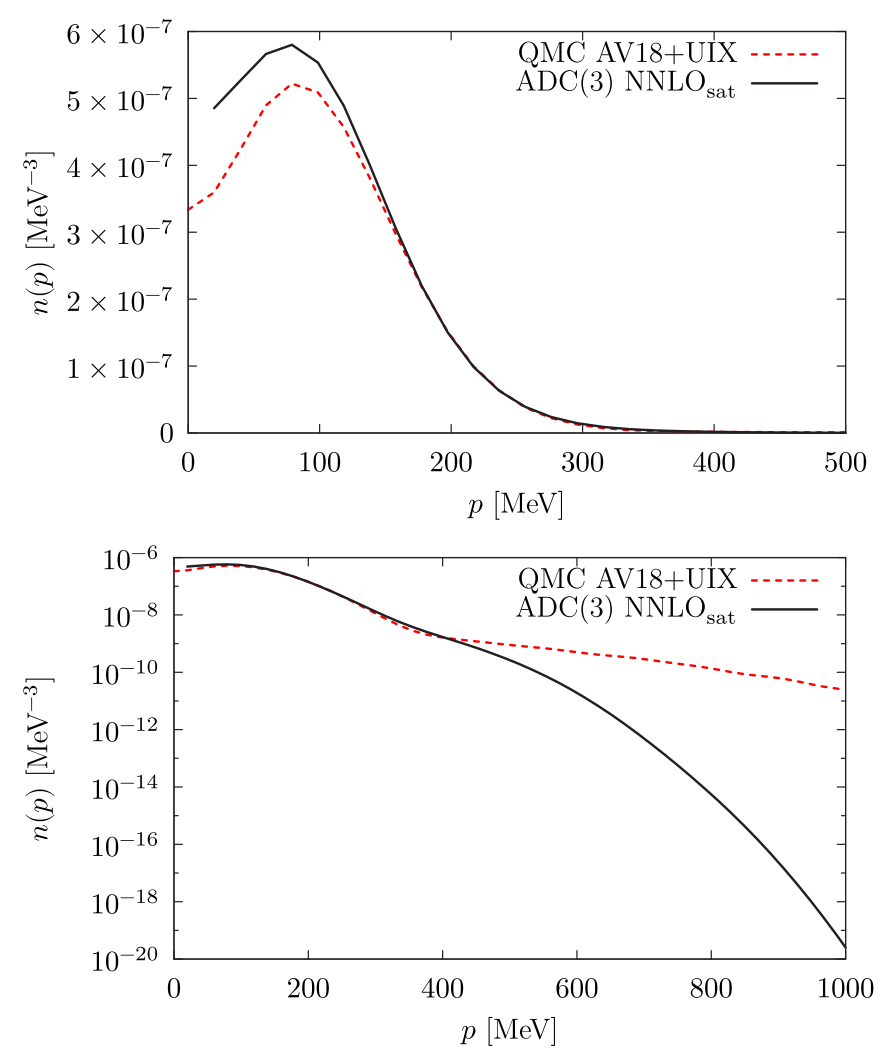

FIG. 8. Computed momentum distributions of ${ }^{16} \mathrm{O}$. The dashed (red) and solid (black) lines are obtained within QMC [41] and SCGF$\mathrm{ADC}(3)$ approaches, respectively. In the lower panel, a logarithmic scale has been used to demonstrate the weak tail at large momenta that arises from the soft chiral interaction adopted in the SCGF-ADC(3) calculation.

in Fig. 8. The differences displayed in the tails of the singlenucleon momentum distributions are clearly visible in the lower panel of Fig. 8, where the logarithmic scale has been used. The dashed (red) line, corresponding to the QMC calculation, is found to be above the SCGF-ADC(3) results for high momenta. This has to be ascribed to the different potentials used. In fact, the $\mathrm{NNLO}_{\text {sat }}$ is much softer than the AV18+UIX potential adopted in the QMC study. The use of a hard potential implies the presence of stronger highmomentum components in the nuclear wave function. While the QMC momentum distribution exhibits a long tail extending to $p>1 \mathrm{GeV}$, the softer potential adopted in our calculations strongly reduce the SCGF-ADC(3) momentum distribution in the high-momentum region. In the upper panel, we observe an enhancement of the SCGF-ADC(3) results with respect to the QMC calculation. This can be understood by recalling that the QMC and SCGF-ADC(3) momentum distribution are normalized to the number of nucleons. In order for the normalization condition to be satisfied, the missing strength in the tails of the $\mathrm{NNLO}_{\text {sat }}$ curve has to be compensated by an enhancement in the low-momentum region.

Figure 9 shows the electron- ${ }^{4} \mathrm{He}$ inclusive doubledifferential cross sections at different values of $E_{e}$ and $\theta_{e}$. The curves are obtained from the full SCGF-ADC(3) spectral 

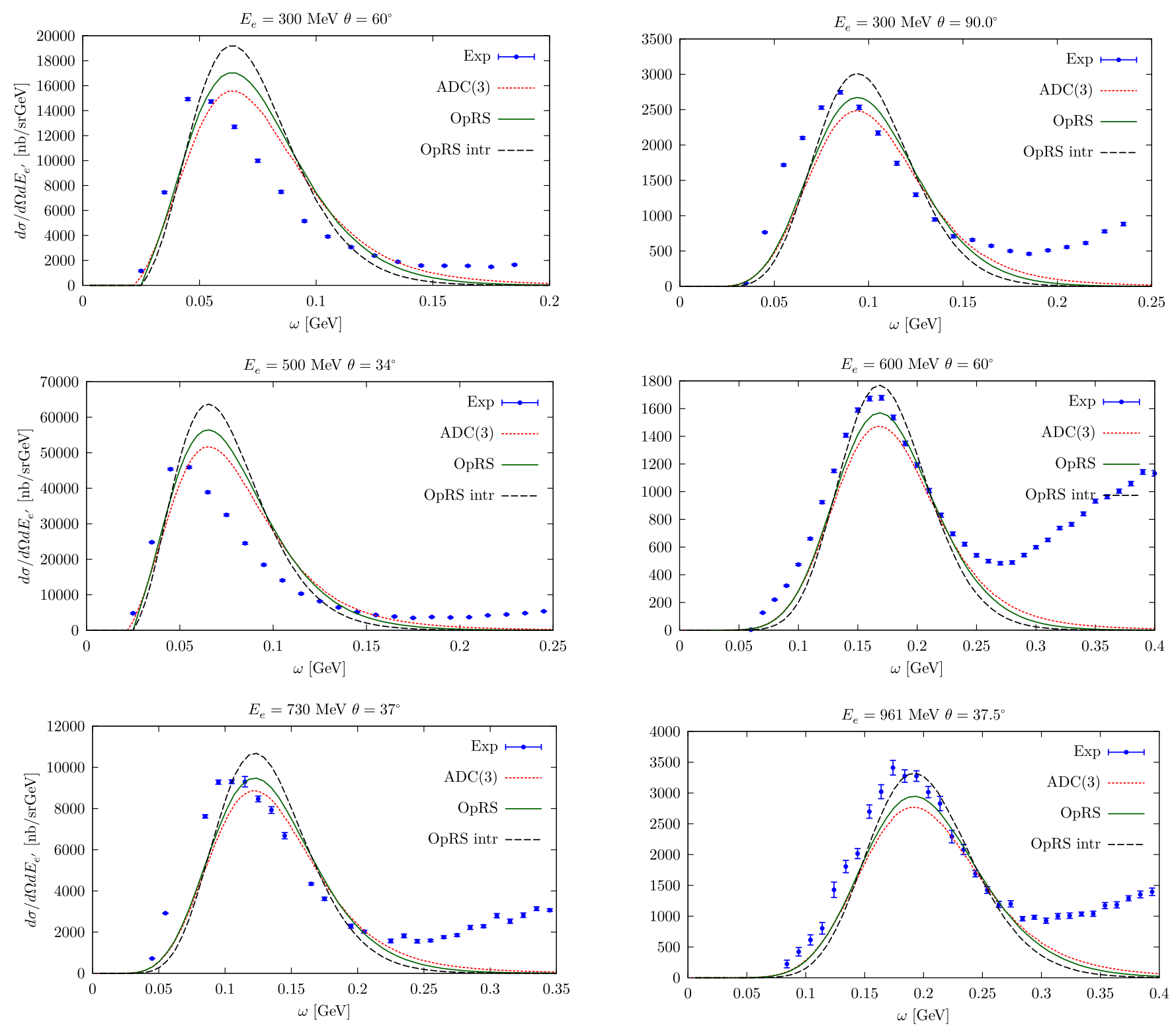

FIG. 9. Double-differential electron- ${ }^{4} \mathrm{He}$ cross sections for different values of incident electron energy and scattering angle. The dotted (red) curve have been obtained using the SCGF-ADC(3) propagator while the solid (green) and dashed (black) line corresponds to the total and the intrinsic OpRS results, respectively. The experimental data are taken from Ref. [49].

function, from its OpRS approximation and from the intrinsic OpRS. The SCGF-ADC(3) cross section represented by the dashed (red) line is quenched with respect to the solid (green) line that refers to the uncorrected OpRS. This has to be attributed to the different behavior of the curves displayed in Fig. 7. While the OpRS wave functions are built to reproduce the lowest energy momenta of the ADC(3) propagator-which optimizes the quasiparticle energies and strength near the Fermi surface- this leaves small discrepancies in the singlenucleon momentum distribution. The comparison between the solid (green) and dashed (black) curve clearly shows that the subtraction of the center-of-mass component from the wave function leads to a reduction of the width and an enhancement of the quasielastic peak. Since this strongly affects the cross section in all the kinematical setups that we considered, we applied FSI corrections only to the intrinsic OpRS calculation. In order to do it, we follow the approach outlined in Sec. III, with the difference that the optical potential has been disregarded in the energy-conserving $\delta$ function since to the best of our knowledge neither the ${ }^{3} \mathrm{H}-p$ nor the ${ }^{3} \mathrm{He}-n$ optical potentials are present in the literature. The results are shown in Fig. 10. The convolution of the OpRS cross section with the folding function of Eq. (38) leads to a redistribution of the strength, which quenches the peak and enhances the tails. For $E_{e}=300 \mathrm{MeV}, \theta=60^{\circ}$, and $E_{e}=500 \mathrm{MeV}, \theta=34^{\circ}$ the OpRS intrinsic calculation overestimates the data. Moreover, in all the kinematical configurations under consideration, the position of the quasielastic peak is not correctly reproduced. 



FIG. 10. Same double-differential cross sections as in Fig. 9 but only for the intrinsic OpRS calculation. The solid (black) line corresponds to neglecting FSI, while the dashed (green) one has been obtained including FSI corrections.

This is likely to be ascribed to the approximate procedure we adopted to account for FSI effects; i.e., we neglected the real part of the optical potential. Its inclusion would shift the cross section toward lower values of $\omega$, possibly improving the agreement with the experimental data.

In Fig. 11, we compare the experimental data of the inclusive double-differential electron- ${ }^{16} \mathrm{O}$ cross sections as computed from the fully correlated SCGF-ADC(3) spectral function. In the dashed (green) curve, FSI effects have been implemented in full, yielding a very nice agreement with the data. In particular, the inclusion of the real part of the optical potential in the final-state nucleon energy shifts the cross sections toward lower values of $\omega$ and the quasielastic-peak position is correctly reproduced.

\section{CONCLUSIONS}

We used the ab initio SCGF approach to compute the singleparticle propagators of closed shell ${ }^{4} \mathrm{He}$ and ${ }^{16} \mathrm{O}$ nuclei. The calculations were based on the the $\mathrm{NNLO}_{\text {sat }}$ chiral interaction since this is capable of describing simultaneously binding energies and nuclear radii of medium-mass nuclei. We have gauged the residual center-of-mass contribution to the ${ }^{4} \mathrm{He}$ wave function by developing a MMC algorithm which exploits the OpRS single-particle propagator. The resulting intrinsic charge density in ${ }^{4} \mathrm{He}$ has been computed and compared with both the QMC calculation of Ref. [41] and the experimental data. The subtraction of the center-of-mass contribution turns out to be crucial in order to obtain correct predictions for this light nucleus. The same pattern has also been observed 

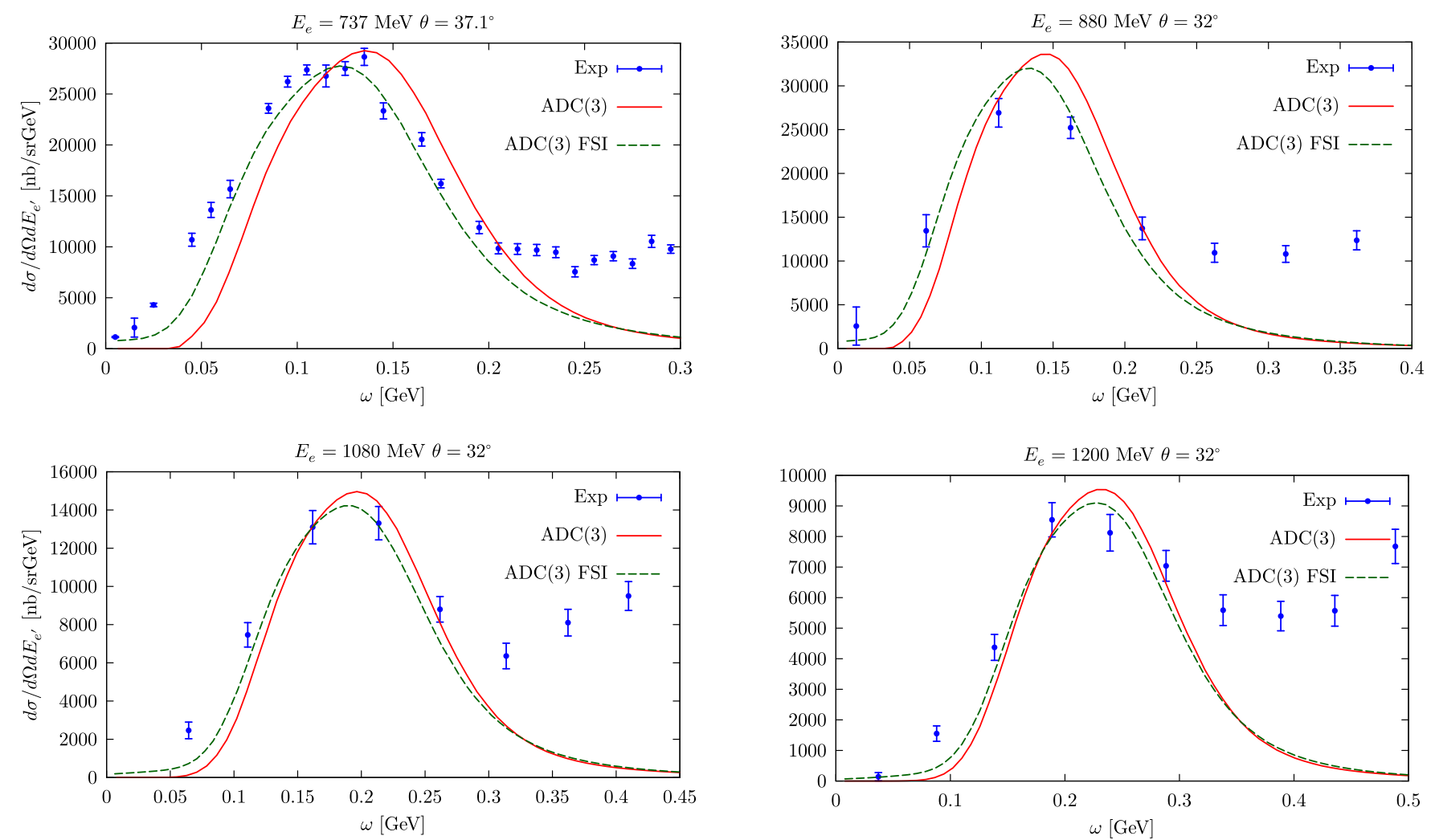

FIG. 11. Double-differential electron- ${ }^{16} \mathrm{O}$ cross sections for different values of incident electron energy and scattering angle. The solid (red) line corresponds to the SCGF-ADC(3) results and the dashed (green) one has been obtained by including FSI corrections. The experimental data are taken from Ref. [49].

in the single-nucleon momentum distribution; while the total OpRS sizably underestimates the QMC calculation, a very nice agreement is found between the QMC and the results from the intrinsic OpRS.

In the analysis of the charge density in ${ }^{16} \mathrm{O}$, the full SCGF-ADC(3) calculation has been compared with the experimental curve. Since the radius of this nucleus has been used to fit the $\mathrm{NNLO}_{\text {sat }}$ potential, the nearly perfect agreement with the empirical charge density is not surprising. However, the very good comparison with experimental cross sections corroborates the choice of the interaction for future studies of lepton-nucleus scattering. The origin of the discrepancies between the single-nucleon momentum distributions obtained from SCGF-ADC(3) and QMC approaches has to be attributed to the softness of the $\mathrm{NNLO}_{\text {sat }}$ interaction. Although the two approaches provide very similar results in the region of low and moderate momentum, the use of a hard potential, such as AV18+UIX, implies a stronger nuclear interaction between large momenta. This manifests itself in the appearance of veryhigh-momentum tails in the momentum distribution which are not as pronounced for the $\mathrm{NNLO}_{\text {sat }}$ chiral force.

We employed the IA approach to perform the calculation of inclusive electromagnetic cross sections, which exploits SCGF spectral functions. The electron- ${ }^{4} \mathrm{He}$ double-differential cross section corresponding to the intrinsic OpRS wave function sizably differs from the OpRS in which the contamination of the center of mass is still present. This indicates that the spurious effect of the center of mass cannot be neglected in light nuclei. For this reason, we restricted the discussion of FSI effects to the sole OpRS intrinsic calculations. We observed that the convolution with the folding function of Refs. [19,20] yields a redistribution of the strength of the cross section. However, disregarding the real part of the optical potential in the energy-conserving $\delta$ function prevents a good agreement with the data for the different kinematical setup analyzed.

Fully satisfactory results have been obtained for the electron- ${ }^{16} \mathrm{O}$ double differential cross section, where the IA calculation has been supplemented by FSI. Our findings indicate that the SCGF approach provides accurate predictions for medium-mass nuclei and their interaction with an electron probe. The extension to the electroweak sector will be the subject of a future work. Moreover, exploiting the Gorkov formalism, we will be able to provide valuable results for open-shell nuclei [50], which will be crucial in the data analysis of future neutrino experiments, such as DUNE.

\section{ACKNOWLEDGMENTS}

We gratefully thank O. Benhar, D. Lonardoni, and J. Lynn for many illuminating discussions and P. Navrátil for providing the matrix elements of $\mathrm{NNLO}_{\text {sat }}$ interaction. We are deeply indebted to P. Massella and A. Lovato for performing the calculation of the OpRS intrinsic wave function of ${ }^{16} \mathrm{O}$. This research has been supported by the Centro Nazionale delle Ricerche (CNR) and the Royal Society under the CNR-Royal Society International Fellowship Scheme No. NF161046 and 
by the United Kingdom Science and Technology Facilities Council (STFC) under Grants No. ST/P005314/1 and No. ST/L005816/1. Calculations were performed at the DiRAC
Complexity system at the University of Leicester (BIS National E-infrastructure Capital Grant No. ST/K000373/1 and STFC Grant No. ST/K0003259/1).
[1] R. Gran, E. J. Jeon, E. Aliu, S. Andringa, S. Aoki, J. Argyriades, K. Asakura, R. Ashie, F. Berghaus, H. Berns et al. (K2K Collaboration), Phys. Rev. D 74, 052002 (2006).

[2] MiniBooNE Collaboration, A. A. Aguilar-Arevalo, A. O. Bazarko, S. J. Brice, B. C. Brown, L. Bugel, J. Cao, L. Coney, J. M. Conrad, D. C. Cox, A. Curioni et al., Phys. Rev. Lett. 100, 032301 (2008).

[3] NOMAD Collaboration, V. Lyubushkin, B. Popov, J. J. Kim, L. Camilleri, J.-M. Levy, M. Mezzetto, D. Naumov, S. Alekhin, P. Astier, D. Autiero et al., Eur. Phys. J. C 63, 355 (2009).

[4] W. H. Dickhoff and C. Barbieri, Prog. Part. Nucl. Phys. 52, 377 (2004).

[5] C. Barbieri and W. H. Dickhoff, Phys. Rev. C 68, 014311 (2003).

[6] C. Barbieri, Phys. Lett. B 643, 268 (2006).

[7] C. Barbieri, D. Van Neck, and W. H. Dickhoff, Phys. Rev. A 76, 052503 (2007).

[8] C. Barbieri and M. Hjorth-Jensen, Phys. Rev. C 79, 064313 (2009).

[9] A. Cipollone, C. Barbieri, and P. Navrátil, Phys. Rev. Lett. 111, 062501 (2013).

[10] V. Soma, T. Duguet, and C. Barbieri, Phys. Rev. C 84, 064317 (2011).

[11] V. Soma, C. Barbieri, and T. Duguet, Phys. Rev. C 87, 011303 (2013).

[12] V. Soma, C. Barbieri, and T. Duguet, Phys. Rev. C 89, 024323 (2014).

[13] A. Carbone, A. Cipollone, C. Barbieri, A. Rios, and A. Polls, Phys. Rev. C 88, 054326 (2013).

[14] A. Carbone, A. Rios, and A. Polls, Phys. Rev. C 90, 054322 (2014).

[15] A. Ekström, G. R. Jansen, K. A. Wendt, G. Hagen, T. Papenbrock, B. D. Carlsson, C. Forssén, M. Hjorth-Jensen, P. Navrátil, and W. Nazarewicz, Phys. Rev. C 91, 051301(R) (2015).

[16] G. Hagen, T. Papenbrock, and D. J. Dean, Phys. Rev. Lett. 103, 062503 (2009).

[17] O. Benhar, N. Farina, H. Nakamura, M. Sakuda, and R. Seki, Phys. Rev. D 72, 053005 (2005).

[18] O. Benhar, D. Day, and I. Sick, Rev. Mod. Phys. 80, 189 (2008).

[19] A. M. Ankowski, O. Benhar, and M. Sakuda, Phys. Rev. D 91, 033005 (2015).

[20] O. Benhar, A. Fabrocini, S. Fantoni, G. A. Miller, V. R. Pandharipande, and I. Sick, Phys. Rev. C 44, 2328 (1991).

[21] W. H. Dickhoff and D. Van Neck, Many-Body Theory Exposed!, 2nd ed. (World Scientific, London, 2008).

[22] J. Schirmer, L. S. Cederbaum, and O. Walter, Phys. Rev. A 28, 1237 (1983).

[23] C. Barbieri and A. Carbone, Self-consistent Green's function approaches, in An Advanced Course in Computational Nuclear Physics: Bridging the Scales from Quarks to Neutron Stars, Lecture Notes in Physics Vol. 936, edited by M. Hjorth-Jensen, M. P. Lombardo, and U. van Kolck (Springer International, Cham, 2017), Chap. 11, pp. 571-644.
[24] G. Hagen, T. Papenbrock, D. J. Dean, A. Schwenk, A. Nogga, M. Wloch, and P. Piecuch, Phys. Rev. C 76, 034302 (2007).

[25] R. Roth, S. Binder, K. Vobig, A. Calci, J. Langhammer, and P. Navratil, Phys. Rev. Lett. 109, 052501 (2012).

[26] C. Barbieri, J. Phys. Conf. Ser. 529, 012005 (2014).

[27] A. Cipollone, C. Barbieri, and P. Navrátil, Phys. Rev. C 92, 014306 (2015).

[28] F. Raimondi and C. Barbieri, Phys. Rev. C 97, 054308 (2018).

[29] A. Rios, A. Carbone, and A. Polls, Phys. Rev. C 96, 014003 (2017).

[30] J. Carlson, S. Gandolfi, F. Pederiva, S. C. Pieper, R. Schiavilla, K. E. Schmidt, and R. B. Wiringa, Rev. Mod. Phys. 87, 1067 (2015).

[31] N. Metropolis, A. W. Rosenbluth, M. N. Rosenbluth, A. H. Teller, and E. Teller, J. Chem. Phys. 21, 1087 (1953).

[32] K. E. Schmidt and S. Fantoni, Phys. Lett. B 446, 99 (1999).

[33] S. C. Pieper, R. B. Wiringa, and V. R. Pandharipande, Phys. Rev. Lett. 64, 364 (1990).

[34] I. Brida, S. C. Pieper, and R. B. Wiringa, Phys. Rev. C 84, 024319 (2011).

[35] S. Pastore, S. C. Pieper, R. Schiavilla, and R. B. Wiringa, Phys. Rev. C 87, 035503 (2013).

[36] O. Benhar, P. Huber, C. Mariani, and D. Meloni, Phys. Rep. 700, 1 (2017)

[37] E. D. Cooper, S. Hama, B. C. Clark, and R. L. Mercer, Phys. Rev. C 47, 297 (1993).

[38] R. F. Garcia Ruiz, M. L. Bissell, K. Blaum, A. Ekström, N. Frömmgen, G. Hagen, M. Hammen, K. Hebeler, J. D. Holt, G. R. Jansen et al., Nat. Phys. 12, 594 (2016).

[39] V. Lapoux, V. Somá, C. Barbieri, H. Hergert, J. D. Holt, and S. Stroberg, Phys. Rev. Lett. 117, 052501 (2016).

[40] H. De Vries, C. W. De Jager, and C. De Vries, At. Data Nucl. Data Tables 36, 495 (1987).

[41] D. Lonardoni, A. Lovato, S. C. Pieper, and R. B. Wiringa, Phys. Rev. C 96, 024326 (2017).

[42] L. E. Marcucci, F. Gross, M. T. Pena, M. Piarulli, R. Schiavilla, I. Sick, A. Stadler, J. W. Van Orden, and M. Viviani, J. Phys. G 43, 023002 (2016).

[43] D. Lonardoni, S. Gandolfi, J. E. Lynn, C. Petrie, J. Carlson, K. E. Schmidt, and A. Schwenk, Phys. Rev. C 97, 044318 (2018).

[44] J. E. Lynn, I. Tews, J. Carlson, S. Gandolfi, A. Gezerlis, K. E. Schmidt, and A. Schwenk, Phys. Rev. C 96, 054007 (2017).

[45] R. F. Frosch, J. S. McCarthy, R. E. Rand, and M. R. Yearian, Phys. Rev. 160, 874 (1967).

[46] J. S. McCarthy, I. Sick, and R. R. Whitney, Phys. Rev. C 15, 1396 (1977).

[47] R. G. Arnold et al., Phys. Rev. Lett. 40, 1429 (1978).

[48] C. R. Ottermann, G. Kobschall, K. Maurer, K. Rohrich, C. Schmitt, and V. H. Walther, Nucl. Phys. A 436, 688 (1985)

[49] O. Benhar, D. Day, and I. Sick, arXiv:nucl-ex/0603032 [nuclex].

[50] V. Somà, A. Cipollone, C. Barbieri, P. Navrátil, and T. Duguet, Phys. Rev. C 89, 061301(R) (2014). 